\title{
Cuidados paliativos en la época de infección por SARS-CoV-2/COVID-19
}

\section{Palliative Care in Times of SARS-CoV-2/COVID-19 Infection}

Recibido: 09 Febrero 2021 | Aceptado: 28 Abril 2021

\begin{abstract}
Carlos José Ariza Galindo a
Unidad de Geriatría, Hospital Universitario San Ignacio, Bogotá, Colombia. Instituto de Envejecimiento, Pontificia Universidad Javeriana, Bogotá, Colombia. Facultad de Medicina, Departamento de Medicina Interna, Pontificia Universidad Javeriana, Bogotá, Colombia

ORCID: https://orcid.org/0000-0002-2502-0337 Ángela Patricia Castiblanco Bautista Facultad de Medicina, Departamento de Medicina Interna. Pontificia Universidad Javeriana, Bogotá, Colombia. Centro de Atención Integral en Cuidados Paliativos, Hospital Universitario San Ignacio, Bogotá, Colombia ORCID: https://orcid.org/0000-0002-8297-3812
\end{abstract}

a Autor de correspondencia: arizacarlos@javeriana.edu.co

Cómo citar: Ariza Galindo CJ, Castiblanco Bautista ÁP. Cuidados paliativos en la época de infección SARS-CoV-2/COVID-19. Univ. Med. 2021;62(3). ht tps://doi.org/10.11144/Javeriana.umed62-3.cpis

\section{RESUMEN}

La nueva pandemia del coronavirus 2019 (COVID-19) está generando desafíos en los sistemas de salud mundiales y planteando dilemas éticos que derivan tanto en la adecuación terapéutica de cada individuo como en la toma de decisiones en contexto de recursos limitados, donde la capacidad de brindar atención médica es insuficiente, así como en aquellos pacientes con múltiples comorbilidades o enfermedad avanzada sin voluntades anticipadas respecto a las medidas de reanimación e intervenciones invasivas como intubación orotraqueal, niveles de asistencia (unidad de cuidado intensivo, sala general, domicilio) y sus preferencias de atención al final de vida. Así mismo, ha evidenciado la necesidad universal del acceso a los cuidados paliativos, al alivio de síntomas y a la importancia de la capacitación del personal de salud en cuidados paliativos en los centros y niveles de atención en salud.

Palabras clave

cuidados paliativos; medicina paliativa; SARS-CoV; COVID19; toma de decisiones.

\section{ABSTRACT}

The new 2019 coronavirus pandemic (COVID-19) is generating challenges in health systems worldwide and posing ethical dilemmas that derive both in the therapeutic adequacy of each individual and in decision-making in the context of limited resources where the capacity of providing medical care is insufficient, as well as in those patients with multiple comorbidities or advanced disease without advance directives regarding resuscitation measures and invasive interventions such as orotracheal intubation, levels of care (ICU, general ward, home) and their care preferences at the end of life. Likewise, it has evidenced the universal need for access to palliative care, symptom relief, and the importance of training health personnel in palliative care at health centers and levels of care.

Keywords

Palliative care; Palliative Medicine; SARS-CoV; COVID19; decision making. 


\section{Introducción}

En el transcurso de diciembre de 2019, en la ciudad de Wuhan, en la provincia de Hubei (China), las autoridades sanitarias identificaron un grupo de casos de neumonía de etiología desconocida (1). La presentación clínica incluyó fiebre, signos radiológicos (insuficiencia pulmonar con opacidades de ocupación alveolar bilateral-patrón de vidrio esmerilado) o signos de dificultad respiratoria aguda, conteo de leucocitos normales o disminuidos y dificultad para resolver el cuadro clínico después de 3 a 5 días de tratamiento con antibióticos. Se observaron vínculos entre los casos índice y el mercado de mariscos del sur de China (2). Con la posibilidad de un nuevo brote de zoonosis o síndrome respiratorio agudo severo (SARS) en mente, se emprendieron investigaciones que desde entonces han identificado un nuevo coronavirus, denominado el coronavirus del síndrome respiratorio agudo severo o grave 2 (SARS-CoV-2, por sus siglas en inglés). A pesar de los titulares dramáticos en la prensa internacional, el brote se ha caracterizado por una respuesta científica extraordinariamente rápida y efectiva, con identificación del patógeno, publicación de su genoma y desarrollo de diagnósticos altamente específicos a las pocas semanas de la detección inicial del caso (3).

La pandemia de la COVID-19 también ha traído a la superficie una serie de problemas sociales y de salud, entre ellos la necesidad urgente de acceso universal a los cuidados paliativos y el alivio de los síntomas. A medida que las tasas de infección y mortalidad continúan aumentando, todos los sistemas de salud están viendo una mayor dependencia de las contribuciones de los servicios de cuidados paliativos.

En medio de la situación de la COVID-19, los cuidados paliativos son esenciales para abordar adecuadamente el dolor y las exacerbaciones de los síntomas, la planificación de la atención anticipada y los objetivos de las discusiones sobre la atención de pacientes con COVID-19 positivo, que enfrentan una descompensación rápida; así mismo, para intervenir en dilemas éticos con respecto a las decisiones al final de la vida, retiro del tratamiento, identificación de duelo anticipado para familiares y cuidadores y el manejo de necesidades de atención complejas para pacientes de alto riesgo, particularmente personas mayores o aquellos con comorbilidades subyacentes e inmunocomprometidos (4).

Históricamente, los cuidados paliativos se han enfocado en aliviar el sufrimiento al final de la vida, lo cual continúa siendo, hasta el momento, la mejor práctica; sin embargo, se implementa cada vez más temprano en las trayectorias de la vida cuando se amenazan las condiciones de salud (5). Una definición reciente basada en consenso define los cuidados paliativos como "la atención holística activa de personas de todas las edades con graves sufrimientos relacionados con la salud debido a enfermedades avanzadas y especialmente a aquellos que están cerca del final de la vida" (6). Esta filosofía y enfoque de la atención busca mejorar la calidad de vida de los pacientes, sus familias y sus cuidadores.

El objetivo de este artículo se centra en describir el impacto de la pandemia en los servicios de cuidados paliativos, así como la toma de decisiones éticas difíciles en los servicios de salud con diferentes niveles de atención.

\section{Impacto de la pandemia}

Para los países de bajos y medianos ingresos, la pandemia de la COVID-19 ha sido aún más grave que en los países de altos ingresos (7). Se ha observado que en estos lugares las personas con COVID-19 grave no tienen posibilidad de acceder a los servicios de salud sufran y mueran en el hogar, donde podrían ser asistidos por familiares sin elementos de protección personal y sin acceso a recursos de cuidados paliativos. Asî mismo, aquellos que logran acceder a los servicios de salud se encuentran con recursos limitados de cuidados intensivos o falta de dispositivos médicos avanzados para su atención.

Durante esta pandemia, la intervención por los servicios de cuidados paliativos esenciales al final de la vida, incluido el apoyo de duelo, será limitado ante las altas demandas en todos 
los países $(8,9)$. Las medidas de distanciamiento físico en busca de disminuir la transmisión de la enfermedad significan escenarios en los cuales los pacientes con diagnóstico de COVID-19, generalmente, no tendrán junto a ellos la compañía de sus seres queridos o no se podrán despedir ni realizar rituales tradicionales de duelo, lo que dificulta aún más esta situación de sufrimiento para el paciente y su familia $(9,10)$.

\section{Toma de decisiones éticas en los servicios de salud}

Durante la atención clínica de los pacientes con infección por COVID-19, el cuidado es fundamental para la atención, por lo que todas las intervenciones deben tener en cuenta los derechos y preferencias de cada persona, en contraste con los deberes de los profesionales, quienes, dentro de una situación de emergencia como la que se está viviendo actualmente, no pueden perder de vista que los derechos y preferencias individuales de las personas pudieran verse reducidos sobre el establecimiento del bien general. Es por esto por lo que se debe responder siempre a los principios de la bioética (justicia, beneficencia, no maleficencia y autonomía) para la atención de pacientes durante la pandemia por SARS-CoV-2/ COVID-19.

Un marco éticamente sólido para la atención de la salud durante las emergencias de salud pública debe equilibrar el deber de atención centrado en el paciente, el enfoque de la ética clínica en condiciones normales con deberes enfocados en el bienestar público para promover la igualdad de las personas y la equidad en la distribución de los riesgos y beneficios en la sociedad: el enfoque de la ética de la salud pública.

El principio ético fundamental para el manejo de pacientes durante las pandemias y otras emergencias de atención médica es que "todos importan por igual", aunque esto no significa que todos sean tratados de la misma manera (11). Es importante que los recursos suficientes estén cercados para pacientes con infección por COVID-19 que tienen intención paliativa, al igual que aquellos con el mismo proceso infeccioso que requieren tratamientos intensivos o de intervención (12).

En el grupo de personas críticamente enfermas por COVID-19, una gran proporción tiende a ser mayor ( $>65$ años) y pueden tener una gran carga de condiciones subyacentes que dificultan su abordaje clínico. El paciente con COVID-19 puede derivar en requerimiento de unidad de cuidados intensivos (UCI) con intubación orotraqueal o sin ella después de considerarse y evaluarse la presencia de comorbilidades, la gravedad de la insuficiencia respiratoria y el tiempo estimado de intubación. Esta situación ha permitido dejar claro que ofrecer un ventilador a un paciente con pocas probabilidades de sobrevida niega la oportunidad de soporte del ventilatorio a otro con mayores posibilidades de supervivencia. Por tanto, en muchos casos, el personal médico debe decidir quién se beneficia o no de estas intervenciones, un dilema similar a los que se enfrentaron en tiempos de guerra.

Ante esta situación, únicamente el criterio de gravedad del afectado no es suficiente, ya que la COVID-19 puede presentar una rápida evolución tórpida, lo que obliga a los médicos a una toma de decisiones apresurada y mediada por la situación del momento (13).

En países como Italia, España y Estados Unidos, que se encuentran entre los más afectados por la pandemia, la disponibilidad limitada de ventiladores y camas hospitalarias en comparación con el número creciente de casos llevó en un momento de la pandemia al establecimiento de un límite de edad con respecto a la provisión de soporte ventilatorio al paciente (8). A partir de este tipo de situaciones, se recomienda, en aras de resolver más eficazmente estos dilemas éticos, que el personal de las UCI debe ser suficiente, contar con un apoyo físico y psicológico, además de estar bien informado y capacitado con respecto a los procedimientos y las decisiones que enfrenten a diario (13). Además, los trabajadores de la salud precisan buscar el asesoramiento de otros equipos con más experiencia cuando estén frente a la toma decisiones cruciales. La cooperación entre 
el personal médico podría conducir a un mejor pronóstico, evaluación y decisión de tratamiento para los pacientes con COVID-19, a fin de mejorar así sus posibilidades de supervivencia.

Retiro de intervenciones y tratamientos no beneficiosos

Los informes de Wuhan y el estado de Washington mostraron altas tasas de mortalidad de hasta un $86 \%$ entre pacientes con COVID-19 que requirieron intubación y ventilación mecánica $(14,15)$. Debido a esta alta tasa de mortalidad, muchos ancianos y pacientes con enfermedades crónicas avanzadas pudieron optar por renunciar a la intubación y ventilación mecánica invasiva; sin embargo, la mayoría de los médicos reciben poca o ninguna capacitación sobre el retiro de tratamiento o intervenciones no beneficiosas y las discusiones acerca de los cuidados al final de la vida (16). En estas situaciones, cuando es probable que las intervenciones invasivas causen más daño e incomodidad que beneficio, debe aplicarse el principio ético de no maleficencia. Se ha descrito que el proceso de informar la posibilidad de disentimiento a intervenciones invasivas ayuda al personal de salud, el paciente y la familia a la toma de decisiones difíciles en momentos críticos, sobre todo cuando son los mismos pacientes quienes hacen énfasis en la calidad de vida que en mantener la longevidad (17). La discusión sobre los objetivos de la atención, idealmente, deberían ocurrir mucho antes de que los pacientes se encuentren severamente enfermos, de tal forma que esto sirva para aliviar la carga de la toma de decisiones de los familiares o el equipo médico $(16,17)$.

\section{Control de sintomas e intervenciones paliativas}

El personal de salud debe recibir capacitación sobre cómo controlar eficazmente síntomas como disnea, náusea, vómito, tos, dolor y delírium en pacientes que no son candidatos a intervenciones invasivas (reanimación cardiopulmonar $\mathrm{O}$ traslado a UCI), con el fin de mantener el confort al final de la vida, dado que su presencia puede generar angustia en el paciente, los familiares y el propio personal de salud $(18,19)$. El control inadecuado de los síntomas puede conducir a intervenciones que aumentan el riesgo de transmisión de enfermedades a trabajadores de la salud, como el uso de ventilación mecánica no invasiva en pacientes con disnea o colocación de restricciones físicas en delírium hiperactivo $(20,21)$. Los esteroides, a menudo, se usan para la paliación de la disnea en pacientes con cáncer avanzado $(22,23)$, y recientemente han sido recomendados para pacientes con COVID-19 y síndrome de dificultad respiratoria aguda con ventilación mecánica (24). Véase tabla 1.

\section{Tabla 1}

Enfoque de cuidados paliativos sugerido para pacientes con COVID19 y comparación con otros enfoques

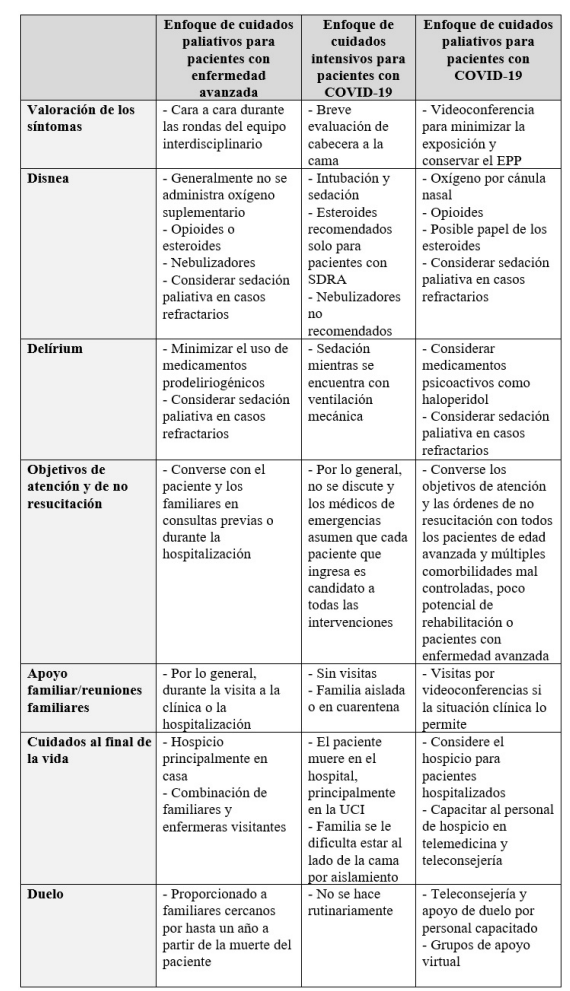

EPP: elementos de protección personal; SDRA: síndrome de dificultad respiratoria aguda Fuente: adaptado de (16)

Otro aspecto importante para la incorporación en la planificación de cuidados paliativos es el apoyo al personal en salud (10). Informes de Italia 
y China mostraron que más de 4800 trabajadores de atención en salud habían sido infectados (9 $\%$ del total de casos de estos reportes) (25-28). Cuidar de los propios colegas y presenciar su muerte puede aumentar aún más la angustia que enfrentan los equipos de atención médica y aumentar el riesgo de agotamiento.

\section{Muerte y soporte en el proceso de duelo}

Se debe garantizar que todas las personas con infección por la COVID-19 que se encuentren en proceso de final de vida reciban atención por parte de un equipo interdisciplinario que incluya cuidados paliativos de alta calidad, especialmente dada su alta carga de síntomas (disnea, ansiedad, delírium, etc.) (29), ya que pueden surgir varios problemas durante el proceso de muerte y duelo en las personas afectadas por COVID-19. Morir solo en un entorno de UCI es difícil para los pacientes y sus seres queridos. Los familiares sobrevivientes a los que no se les permite estar con sus seres queridos durante este momento crítico corren un alto riesgo de sufrimiento. Después del proceso de muerte, los cuerpos de las personas fallecidas deben ser manejados de forma especial para prevenir la propagación de la infección (30). Todos estos requisitos complican aún más el proceso de duelo para los miembros de la familia que, probablemente, se encuentran en cuarentena o a la espera de resultados de pruebas para definir si están o no infectados, lo que incrementa la angustia y la agonía. Las intervenciones para brindar asesoramiento sobre duelo deben incorporar métodos basados en la virtualidad, al igual que contacto con las familias afectadas mediante grupos virtuales de apoyo, que pueden ayudarlos en su proceso de duelo a compartir experiencias y encontrar respaldo emocional (31-33). Dispositivos electrónicos son la única forma de garantizar la aproximación y acompañamiento en el lecho de muerte de los pacientes.

\section{Conclusión}

Durante lo transcurrido de la pandemia por COVID-19 se ha observado un tendencia de la situación a superar la capacidad de los sistemas de salud en algunos países del mundo, por lo que estamos convencidos de la necesidad de integrar los cuidados paliativos en la planificación de la atención en salud en todos los escenarios posibles. Se ha evidenciado una necesidad inmediata de capacitación a los profesionales en salud sobre los principios de los cuidados paliativos y cómo clasificar a los pacientes cuando los recursos son escasos, además de la necesidad de métodos alternativos para el acceso a los cuidados paliativos, por ejemplo, telemedicina, asesoramiento psicológico y grupos de apoyo virtuales.

\section{Conflicto de intereses}

Los autores declaran no tener conflictos de intereses para esta publicación.

\section{Referencias}

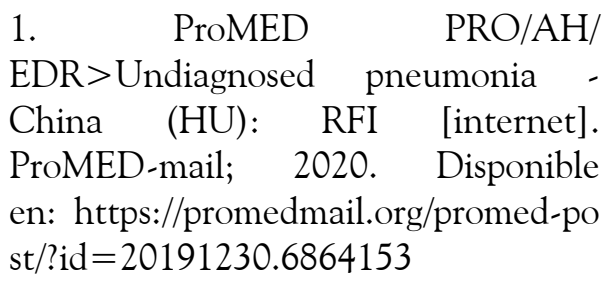

2. Wiersinga WJ, Rhodes A, Cheng AC, Peacock SJ, Prescott HC. Pathophysiology, transmission, diagnosis, and treatment of coronavirus disease 2019 (COVID-19): a review. JAMA. 2020 Jul 10. https://doi.org/10. 1001/jama.2020.12839

3. Lake MA. What we know so far: COVID-19 current clinical knowledge and research. Clin Med. 2020 Mar;20(2):124-7. https://doi.org/ 10.7861/clinmed.2019-coron

4. Rosa WE, Davidson

PM. Coronavirus disease 2019 
(COVID-19): strengthening our resolve to achieve universal palliative care. Int Nurs Rev. 2020 Jun;67(2):160-3. https://doi.org/10.11 11/inr.12592

5. World Health Organization. Strengthening of palliative care as a component of integrated treatment throughout the life course. J Pain Palliat Care Pharmacother. 2014 Jun;28(2):130-4. https://doi.org/10.31 09/15360288.2014.911801

6. Radbruch L, De Lima L, Knaul F, Wenk R, Ali Z, Bhatnaghar S, et al. Redefining palliative carea new consensus-based definition. J Pain Symptom Manage. 2020 May;60(4):754-64. https://doi.org/10.1 016/j.jpainsymman.2020.04.027

7. Radbruch L, Knaul FM, de Lima L, de Joncheere C, Bhadelia A. The key role of palliative care in response to the COVID-19 tsunami of suffering. Lancet (London, England). 2020 May 9;395(10235):1467-9. https://doi.org/1 0.1016/S0140-6736(20)30964-8

8. Rosenbaum L. Facing Covid-19 in Italy - ethics, logistics, and therapeutics on the epidemic's front line. N Engl J Med. 2020 Mar 18;382(20):1873-5. https://doi.org/10. 1056/NEJMp2005492

9. Costantini M, Sleeman KE, Peruselli $\mathrm{C}$, Higginson IJ. Response and role of palliative care during the COVID-19 pandemic: a national telephone survey of hospices in Italy. Palliat Med. 2020 Jul;34(7):889-95.

10. Wallace CL, Wladkowski SP, Gibson A, White P. Grief During the COVID-19 pandemic: considerations for palliative care providers. J Pain Symptom Manage. $2020 \mathrm{Jul}$ 1;60(1):e70-6. https://doi.org/10.1016/ j.jpainsymman.2020.04.012
11. Cabinet Office and Department

of Health. Responding to pandemic influenza: the ethical framework for policy and planning [internet]. London: Department of Health; 2007. Disponible en: https://www.hsj.co.uk/swine-flu/res ponding-to-pandemic-influenza-the-et hical-framework-for-policy-and-planni ng/5005219.article

12. Davies A, Hayes J. Palliative care in the context of a pandemic: similar but different. Clin Med. 2020 Apr;20(3):274-7.

13. Karampelias V, Spanidis Y, Roussakou E. Ethical issues in intensive care units during the COVID-19 pandemic. Indian Soc Crit Care Med. 2020 Sep;24(9):855-6.

14. Yang $\mathrm{X}, \mathrm{Yu} \mathrm{Y}, \mathrm{Xu} \mathrm{J}$, Shu H, Xia J, Liu $H$, et al. Clinical course and outcomes of critically ill patients with SARS-CoV-2 pneumonia in Wuhan, China: a singlecentered, retrospective, observational study. Lancet Respir Med. 2020 May;8(5):475-81.

15. Bhatraju PK, Ghassemieh BJ, Nichols M, Kim R, Jerome KR, Nalla $\mathrm{AK}$, et al. Covid-19 in critically ill patients in the seattle region case series. N Engl J Med. 2020 May;382(21):2012-22.

16. Fadul N, Elsayem AF, Bruera E. Integration of palliative care into COVID-19 pandemic planning. BMJ Support Palliat Care. 2020 Jun. https://doi.org/10.1136/bmjspcare $-2020-002364$

17. Curtis JR, Kross EK, Stapleton RD. The importance of addressing advance care planning and decisions about do-not-resuscitate orders during novel coronavirus 2019 (COVID-19). JAMA. 2020 Mar;323(18):1771-2. htt ps://doi.org/10.1001/jama.2020.4894 
18. Najjar N, Davis LW, Beck-Coon $\mathrm{K}$, Carney Doebbeling C. Compassion fatigue: a review of the research to date and relevance to cancer-care providers. J Health Psychol. 2009 Mar;14(2):267-77.

19. Portoghese I, Galletta M, Larkin P, Sardo S, Campagna M, Finco G, et al. Compassion fatigue, watching patients suffering and emotional display rules among hospice professionals: a daily diary study. BMC Palliat Care. 2020 Feb;19(1):23.

20. Kunz R, Minder M. COVID-19 pandemic: palliative care for elderly and frail patients at home and in residential and nursing homes. Swiss Med Wkly. 2020 Mar;150:w20235.

21. Hendin A, La Rivière CG, Williscroft DM, O'Connor E, Hughes J, Fischer LM. End-of-life care in the emergency department for the patient imminently dying of a highly transmissible acute respiratory infection (such as COVID-19). CJEM. 2020 Mar;1-4.

22. Elsayem A, Bruera E. High-dose corticosteroids for the management of dyspnea in patients with tumor obstruction of the upper airway. Support care cancer Off J Multinatl Assoc Support Care Cancer. 2007 Dec;15(12):1437-9.

23. Hui D, Kilgore $\mathrm{K}$, FrisbeeHume S, Park M, Tsao A, Delgado Guay M, et al. Dexamethasone for dyspnea in cancer patients: a pilot double-blind, randomized, controlled trial. J Pain Symptom Manage. 2016 Jul;52 (1):8-16.e1.

24. Alhazzani W, Møller MH, Arabi YM, Loeb M, Gong MN, Fan E, et al. Surviving sepsis campaign: guidelines on the management of critically ill adults with coronavirus disease 2019 (COVID-19). Crit Care Med. 2020 Jun;48(6):e440-69.
25. Paterlini M. On the front lines of coronavirus: the Italian response to covid-19. BMJ. 2020 Mar;368:m1065.

26. Anelli F, Leoni G, Monaco R, Nume C, Rossi RC, Marinoni G, et al. Italian doctors call for protecting healthcare workers and boosting community surveillance during covid-19 outbreak. BMJ. 2020 Mar 26;368:m1254. https:// doi.org/10.1136/bmj.m1254

27. Ng K, Poon BH, Kiat Puar TH, Shan Quah JL, Loh WJ, Wong YJ, et al. COVID-19 and the risk to health care workers: a case report. Ann Intern Med. 2020 Jun 2;172(11):766-7. https: //doi.org/10.7326/L20-0175

28. Schwartz J, King C-C, Yen M-Y. Protecting health care workers during the COVID-19 coronavirus outbreak: lessons from Taiwan's SARS response. Clin Infect Dis an Off Publ Infect Dis Soc Am. 2020 Mar;71(15):858-60. htt ps://doi.org/10.1093/cid/ciaa255

29. Borasio GD, Gamondi C, Obrist M, Jox R, For The Covid-Task Force Of Palliative Ch. COVID-19: decision making and palliative care. Swiss Med Wkly. 2020 Mar 24;150:w20233. https ://doi.org/10.4414/smw.2020.20233

30. European Centre for Disease Prevention and Control. Considerations related to the safe handling of bodies of deceased persons with suspected or confirmed COVID-19 [internet]. Stockholm: ECDC; 2020. Disponible en: https://www.ecdc.europa.eu/sites/d efault/files/documents/COVID-19-safe -handling-of-bodies-or-persons-dying- $f$ rom-COVID19.pdf

31. Wagner B, Knaevelsrud C, Maercker A. Internet-based cognitivebehavioral therapy for complicated grief: a randomized controlled trial. Death Stud. 2006 Jun;30(5):429-53. 
32. Amstadter AB, Broman-Fulks J, Zinzow $\mathrm{H}$, Ruggiero KJ, Cercone J. Internet-based interventions for traumatic stress-related mental health problems: a review and suggestion for future research. Clin Psychol Rev. 2009 Jul;29(5):410-20.

33. Swartwood RM, Veach PM, Kuhne J, Lee HK, Ji K. Surviving grief: an analysis of the exchange of hope in online grief communities. Omega. 2011;63(2):161-81. 